\title{
Atuação do Cirurgião-Dentista na equipe multidisciplinar em Unidade de Terapia Intensiva: Percepção dos acadêmicos
}

\author{
Performance of the Dental Surgeon in the multidisciplinary team in the Intensive Care Unit:
} Students' perception

Desempeño del Cirujano Dentista en el equipo multidisciplinario de la Unidad de Cuidados Intensivos: Percepción de los estudiantes

\author{
Andreia Medeiros Rodrigues Cardoso \\ ORCID: https://orcid.org/0000-0002-2682-1048 \\ Centro Universitário de João Pessoa, Brasil \\ E-mail: andreiamedeiros29@yahoo.com.br \\ Talita Helen Araújo de Almeida \\ ORCID: https://orcid.org/0000-0002-5736-0109 \\ Centro Universitário de João Pessoa, Brasil \\ E-mail: talita.hellen.araujo@gmail.com \\ Kauana da Silva Andrade \\ ORCID: https://orcid.org/0000-0002-0385-9983 \\ Centro Universitário de João Pessoa, Brasil \\ E-mail: kauanaandrade12@gmail.com \\ Thaynara Cavalcante Moreira Romão \\ ORCID: https://orcid.org/0000-0002-9695-1251 \\ Centro Universitário de João Pessoa, Brasil \\ E-mail: thaynaracavalcante1@gmail.com \\ José Jhenikártery Maia de Oliveira \\ ORCID: https://orcid.org/0000-0003-2915-5607 \\ Centro Universitário de João Pessoa, Brasil \\ E-mail: jhenikarterymaia@gmail.com \\ Maria do Carmo Pessoa Nogueira Serrão \\ ORCID: https://orcid.org/0000-0002-0500-7485 \\ Hospital Metropolitano Dom José Maria Pires, Brasil \\ E-mail: carminha11@hotmail.com \\ Daniel Furtado Silva \\ ORCID: https://orcid.org/0000-0003-3319-2996 \\ Universidade Federal da Paraíba, Brasil \\ E-mail: furtado.ds@gmail.com \\ Fernando Martins Baeder \\ ORCID: https://orcid.org/0000-0001-7101-5689 \\ Universidade Cruzeiro do Sul, Brasil \\ E-mail: fernandobaeder@uol.com.br
}

\begin{abstract}
Resumo
Objetivo: Descrever a percepção dos acadêmicos, do décimo período do curso de Odontologia de duas Instituições de Ensino Superior, sobre a atuação do cirurgião-dentista (CD) em Unidade de Terapia Intensiva (UTI). Metodologia: Trata-se de um estudo observacional transversal, com aplicação de questionário para acadêmicos concluintes do curso de Odontologia, sobre a atuação do CD na UTI, os protocolos de higiene e a relação dos patógenos bucais com doenças sistêmicas. Os dados foram analisados descritivamente e de forma analítica por meio do teste do quiquadrado. Resultados: Dos 71 participantes, 87,3\% afirmaram conhecer sobre a atuação do CD na UTI. Todos os entrevistados julgaram como importante o tema Odontologia Hospitalar e a presença do CD na equipe multidisciplinar, porém 7\% se consideravam aptos para atuar nesta área. Cerca de $87 \%$ dos acadêmicos consideraram a solicitação de exames complementares, prescrição e realização da desinfecção da cavidade bucal como atribuições do $\mathrm{CD}$ em ambiente hospitalar. $\mathrm{O}$ procedimento de prescrição medicamentosa e solicitação de exames complementares foi mais relatado pelos alunos que tiveram aulas sobre Odontologia Hospitalar na graduação ( $\mathrm{p}<0,05)$. Cerca de $62 \%$ dos acadêmicos têm conhecimento sobre os protocolos de higiene bucal que devem ser realizados na UTI e todos os acadêmicos avaliados afirmaram que a doença periodontal tem influência nas patologias sistêmicas. Conclusão: Os acadêmicos consideraram que o CD deve participar da equipe que compõe a UTI, possuíam
\end{abstract}


algumas informações sobre a atuação da Odontologia no ambiente hospitalar, no entanto, poucos se sentem aptos para atuar nesse espaço de trabalho.

Palavras-chave: Estudantes de odontologia; Equipe hospitalar de odontologia; Unidades de Terapia Intensiva; Ensino.

\begin{abstract}
Objective: To describe the perception of academics, from the tenth period of the Dentistry course of two Higher Education Institutions, about the performance of the dentist (CD) in the Intensive Care Unit (ICU). Methodology: This is a cross-sectional observational study, with the application of a questionnaire for graduates of the Dentistry course, about the performance of the DC in the ICU, the hygiene protocols and the relationship of oral pathogens with systemic diseases. The data were analyzed descriptively and analytically using the chi square test. Results: Of the 71 participants, $87.3 \%$ said they knew about the performance of the DC in the ICU. All respondents considered the theme of Hospital Dentistry and the presence of the CD in the multidisciplinary team as important, but $7 \%$ considered themselves able to work in this area. Approximately $87 \%$ of academics considered requesting complementary exams, prescribing and performing disinfection of the oral cavity as assignments of the DC in a hospital environment. The procedure of drug prescription and requesting complementary exams was most reported by students who had classes on Hospital Dentistry at graduation ( $\mathrm{p}<0.05$ ). Approximately $62 \%$ of academics are aware of the oral hygiene protocols that must be performed in the ICU and all evaluated academics stated that periodontal disease has an influence on systemic pathologies. Conclusion: The academics considered that the DC should participate in the team that makes up the ICU, they had some information about the performance of Dentistry in the hospital environment, however, few feel able to work in this work space.
\end{abstract}

Keywords: Students, dental; Dental staff, hospital; Intensive Care Units; Teaching.

\title{
Resumen
}

Objetivo: Describir la percepción de los académicos, del décimo período del curso de Odontología de dos Instituciones de Educación Superior, sobre el desempeño del odontólogo (CD) en la Unidad de Cuidados Intensivos (UCI). Metodología: Se trata de un estudio observacional transversal, con la aplicación de un cuestionario para egresados de la carrera de Odontología, sobre el desempeño de la CD en la UCI, los protocolos de higiene y la relación de patógenos bucales con enfermedades sistémicas. Los datos se analizaron de forma descriptiva y analítica mediante la prueba de chi cuadrado. Resultados: De los 71 participantes, el 87,3\% dijo conocer el desempeño de la CD en la UCI. Todos los encuestados consideraron importante la temática de la Odontología Hospitalaria y la presencia del $\mathrm{CD}$ en el equipo multidisciplinar, pero el $7 \%$ se consideró capaz de trabajar en esta área. Aproximadamente el $87 \%$ de los académicos consideraron solicitar exámenes complementarios, prescribir y realizar la desinfección de la cavidad bucal como asignaciones del CD en un entorno hospitalario. El procedimiento de prescripción de medicamentos y solicitud de exámenes complementarios fue más informado por los estudiantes que tenían clases de Odontología Hospitalaria al egreso ( $\mathrm{p}<0.05$ ). Aproximadamente el $62 \%$ de los académicos conocen los protocolos de higiene bucal que se deben realizar en la UCI y todos los académicos evaluados manifestaron que la enfermedad periodontal influye en las patologías sistémicas. Conclusión: Los académicos consideraron que el DC debe participar en el equipo que conforma la UCI, tenían alguna información sobre el desempeño de la Odontología en el ámbito hospitalario, sin embargo, pocos se sienten capaces de trabajar en este espacio de trabajo.

Palabras clave: Estudiantes de odontología; Personal de odontología en hospital; Unidades de Cuidados Intensivos; Ensenãnza.

\section{Introduçãa}

A Unidade de Terapia Intensiva (UTI) refere-se a atuação multiprofissional direcionada aos pacientes com comprometimento das suas funções vitais, resultando da falha de um ou mais sistemas orgânicos. É considerado o nível mais complexo e avançado da Unidade hospitalar, proporcionando o monitoramento do paciente por 24 horas ao dia (Marshall et al., 2017).

Os pacientes internados em UTI estão, na maioria das vezes, acometidos por alguma patologia grave, onde existe um risco de vida eminente. Neste contexto, patologias bucais atuam como foco de disseminação de agentes patogênicos com efeitos metastático sistêmico, principalmente em pacientes com imunidade comprometida. A prática odontológica em UTI é fundamental para a prevenção e controle de patologias como, por exemplo, as infecções respiratórias (Sampaio-Maia et al., 2016; Ory et al., 2018). 
A Odontologia Hospitalar é definida como a área da Odontologia responsável por cuidar de pacientes em ambiente hospitalar, com enfoque nas estruturas do sistema estomatognático (Morais et al., 2008). Dessa forma, ressalta-se a necessidade de cuidados bucais, principalmente nas primeiras 48 a 72 horas de internação na UTI, pois esse é o período de mudança da microbiota bucal, com predominância de bactérias Gram-negativas, o que pode estar associado a quadros infecciosos como a pneumonia (Amaral et al., 2013).

A Lei Federal Nº 2776/08 torna obrigatória a presença do cirurgião-dentista em UTI de hospital públicos e privados com pacientes internados de médio e grande porte. O objetivo destes profissionais nesses centros é a manutenção da higiene bucal dos pacientes por meio de medidas preventivas e do tratamento de doenças bucais, como, por exemplo, a cárie dentária, a doença periodontal, as estomatites, entre outras. Dessa forma, contribui-se para que tratamento médico não seja interrompido e o paciente se recupere mais rapidamente (Brasil, 2008).

Diante do exposto, o objetivo desse estudo foi descrever a percepção dos acadêmicos de Odontologia sobre a atuação do cirurgião-dentista na equipe multidisciplinar em uma UTI, como também descrever a percepção sobre higiene bucal em UTI e a relação da condição bucal com as complicações sistêmicas.

\section{Metodologia}

Trata-se de um estudo observacional transversal, de natureza quantitativa e com procedimento descritivo, realizado no período de setembro a outubro de 2019. Como descrito por Pereira et al. (2018), a pesquisa quantitativa se caracteriza pela coleta de dados quantitativos ou numéricos, utilizando medições de grandezas obtendo-se números e suas determinadas unidades. A pesquisa foi desenvolvida no curso de Odontologia de duas Instituições de Ensino Superior (IESs), sendo uma pública e a outra privada, localizadas em João Pessoa, na Paraíba.

O universo compreendeu oitenta (80) acadêmicos do décimo período das IESs. A amostra não probabilística e por conveniência, foi constituída por setenta e um (71) concluintes do curso de Odontologia. Foram incluídos graduandos do décimo período do curso de Odontologia das duas IESs. Considerou-se como perda a recusa em participar da pesquisa e os participantes cujos questionários não apresentaram cinquenta (50\%) das respostas.

O instrumento de coleta dos dados foi um questionário, com vinte e oito (28) questões, aplicado aos participantes no local do estudo. $\mathrm{O}$ instrumento foi elaborado pela equipe da pesquisa e as variáveis analisadas contemplam as características da amostra, a percepção dos acadêmicos sobre a atuação do cirurgião-dentista na equipe multidisciplinar, os protocolos de higiene bucal e a relação da condição bucal com as doenças sistêmicas.

A coleta dos dados teve início após apreciação pelo Comitê de Ética em Pesquisa do Centro Universitário de João Pessoa (UNIPÊ), com CAAE 15999019.7.0000.5176. Todos os participantes assinaram o Termo de Consentimento Livre e Esclarecido (TCLE).

Os dados foram analisados descritivamente com medidas de distribuição de medidas absolutas, percentuais, médias, medianas e desvios padrões através do software Statistical Package for the Social Sciences (SPSS), versão 20. Em seguida, realizou-se a análise inferencial com o teste de qui-quadrado, com nível de significância estatística de 5\%, para verificar a associação entre as variáveis dos procedimentos odontológicos e ter cursado sobre a Odontologia Hospitalar em componente curricular.

\section{Resultados}

Dos 80 acadêmicos matriculados no décimo período do curso de Odontologia das duas IESs, 71 participaram da pesquisa, sendo equivalente a uma taxa de resposta de $88,7 \%$. 
A maioria dos acadêmicos eram do sexo feminino 77,5\% (n=55), 50,7\% ( $n=36)$ estavam na faixa etária de 22 a 25 anos. Em relação a renda familiar, 52,9\% $(\mathrm{n}=36)$ apresentavam entre três a quatro salários mínimos e 19,7\% (n=14) possuíam Ensino Superior completo, conforme a Tabela 1.

Tabela 1 - Caraterização da amostra. João Pessoa, 2019.

\begin{tabular}{|c|c|c|}
\hline Variável & $\mathbf{N}$ & $\%$ \\
\hline \multicolumn{3}{|l|}{ Faixa etária } \\
\hline Entre 18 e 21 & 5 & $7 \%$ \\
\hline Entre 22 e 25 & 36 & $50,7 \%$ \\
\hline Entre 26 e 30 & 23 & $32,4 \%$ \\
\hline Acima de 31 & 7 & $9,9 \%$ \\
\hline \multicolumn{3}{|l|}{ Sexo } \\
\hline Feminino & 55 & $77,5 \%$ \\
\hline Masculino & 16 & $22,5 \%$ \\
\hline \multicolumn{3}{|l|}{ Formação Acadêmica } \\
\hline Sim & 14 & $19,7 \%$ \\
\hline Não & 57 & $80,3 \%$ \\
\hline \multicolumn{3}{|l|}{ Qual formação? } \\
\hline Enfermagem & 8 & $57,1 \%$ \\
\hline Administração & 3 & $21,4 \%$ \\
\hline Marketing & 1 & $7,14 \%$ \\
\hline Não responderam & 2 & $14,3 \%$ \\
\hline \multicolumn{3}{|l|}{ Renda Familiar } \\
\hline 1 a 2 salários mínimo & 20 & $29,4 \%$ \\
\hline 3 a 4 salários mínimo & 36 & $52,9 \%$ \\
\hline Acima de 5 salários mínimo & 12 & $17,6 \%$ \\
\hline Não responderam & 3 & $0,1 \%$ \\
\hline
\end{tabular}

Fonte: Dados da pesquisa (2019).

Cerca de 88,7\% (n=63) dos entrevistados não participaram de nenhum projeto de extensão ou pesquisa na área da Odontologia Hospitalar. Todos os participantes declararam como importante a abordagem da temática durante a graduação, enquanto $7 \%(\mathrm{n}=5)$ consideravam-se aptos para atuar em UTI (Tabela 2). 
Tabela 2 - Distribuição da amostra de acordo com o conhecimento e a experiência com a Odontologia Hospitalar. João Pessoa, 2019.

\begin{tabular}{|c|c|c|}
\hline Variável & $\mathbf{N}$ & $\%$ \\
\hline \multicolumn{3}{|l|}{ Trabalho voluntário em hospital } \\
\hline Sim & 19 & $26,8 \%$ \\
\hline Não & 52 & $73,2 \%$ \\
\hline \multicolumn{3}{|c|}{ Participou de projeto de extensão ou pesquisa em Odontologia Hospitalar? } \\
\hline Sim & 8 & $11,3 \%$ \\
\hline Não & 63 & $88,7 \%$ \\
\hline \multicolumn{3}{|c|}{$\begin{array}{l}\text { Algum componente curricular abordou o tema relacionado sobre a atuação do } \\
\text { Cirurgião-Dentista em unidade hospitalar? }\end{array}$} \\
\hline Sim & 62 & $87,3 \%$ \\
\hline Não & 9 & $12,7 \%$ \\
\hline \multicolumn{3}{|l|}{ Qual componente? } \\
\hline Pacientes com Necessidades Especiais & 62 & $87,3 \%$ \\
\hline Não se aplica & 9 & $12,7 \%$ \\
\hline \multicolumn{3}{|c|}{ Acha importante o tema "Odontologia hospitalar" ser abordado na graduação? } \\
\hline Sim & 71 & $100 \%$ \\
\hline \multicolumn{3}{|c|}{ Com a graduação já está apto para atuar em uma UTI? } \\
\hline $\operatorname{Sim}$ & 5 & $7 \%$ \\
\hline Não & 66 & $93 \%$ \\
\hline \multicolumn{3}{|l|}{ Já conheceu alguma UTI? } \\
\hline Sim & 31 & $44,9 \%$ \\
\hline Não & 38 & $55,1 \%$ \\
\hline \multicolumn{3}{|c|}{ O Cirurgião-dentista deve fazer parte da equipe multidisciplinar na UTI? } \\
\hline Sim & 71 & $100 \%$ \\
\hline
\end{tabular}

Fonte: Dados da pesquisa (2019).

A Tabela 3 apresenta a associação entre a percepção dos acadêmicos sobre os procedimentos odontológicos, realizados em ambiente hospitalar, e ter cursado algum componente curricular que abordou sobre a Odontologia hospitalar. Foi observado que 93,5\% (n=58) dos participantes que responderam solicitar exames complementares e realizar prescrições, cursou o componente curricular, com diferença significativa.

No entanto, alguns alunos também relataram a realização de procedimentos como tratamento restaurador protético (21\%), tratamento endodôntico (33\%) e a exodontia de terceiros molares sem foco de infecção (31\%) e a frequência não mostrou diferença significativa de acordo com o fato de ter recebido alguma aula na graduação sobre Odontologia Hospitalar ( $>>0,05)$. 
Tabela 3 - Associação entre as atribuições do cirurgião-dentista, de acordo com a percepção dos acadêmicos, e a abordagem da Odontologia Hospitalar através de componente curricular. João Pessoa, 2019.

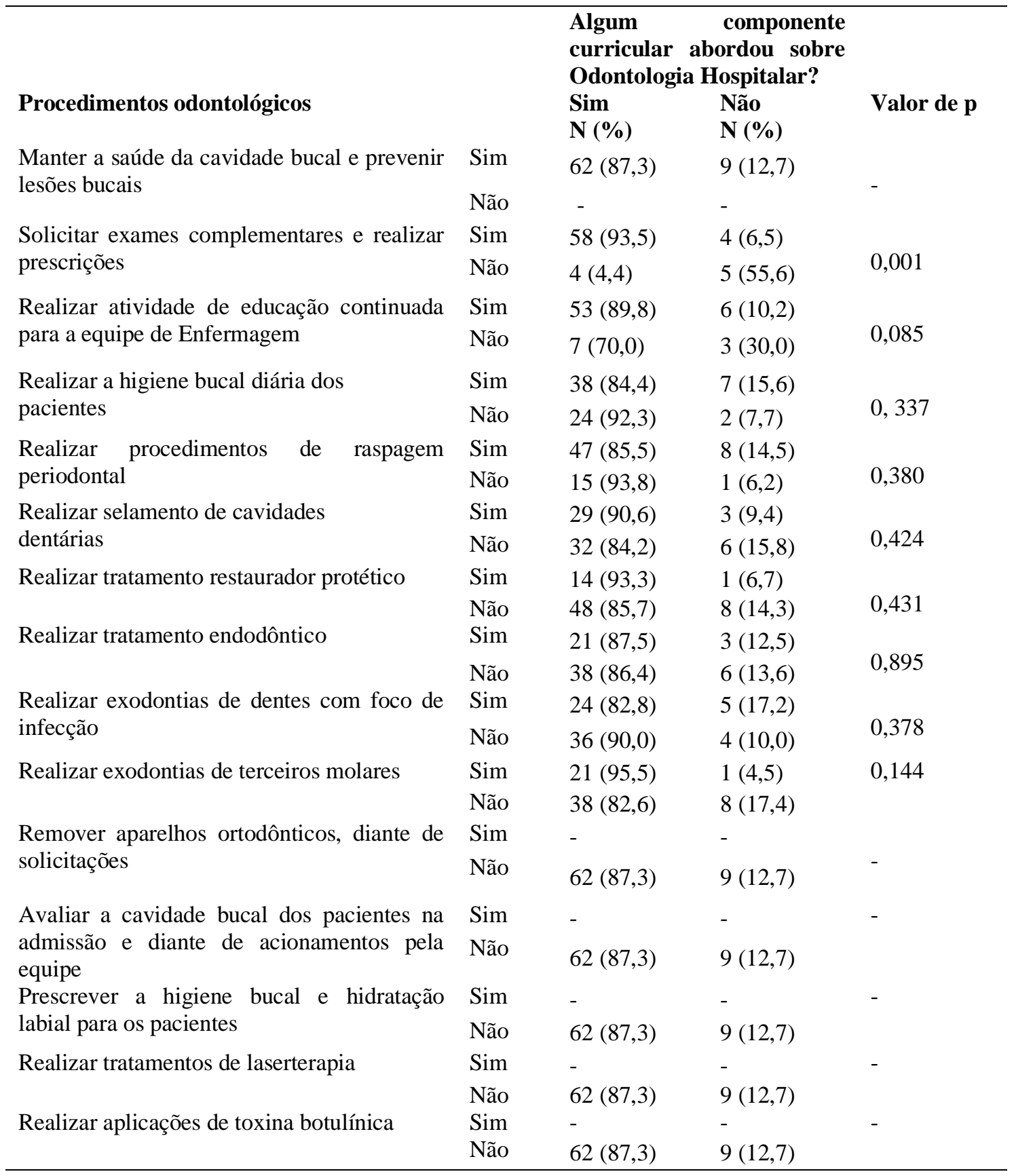

Fonte: Dados da pesquisa (2019).

Em relação ao profissional responsável pela higiene bucal diária dos pacientes internos em UTI, 66,19\% (n=47) dos acadêmicos afirmaram que seria o cirurgião-dentista, e 35,2\% $(n=25)$ atribuiu à equipe de Enfermagem.

Ao serem questionados sobre o conhecimento dos protocolos de higiene bucal que devem ser realizados em UTI, 62\% $(n=44)$ dos acadêmicos responderam que sim, enquanto $38 \%(n=27)$ desconhecem esses protocolos. Em relação aos produtos químicos utilizados nos protocolos de higiene bucal para paciente adulto intubado e interno em UTI, foram citados com 98,5\% $(\mathrm{n}=70)$ o Digluconato de Clorexidina a $0,12 \%$, o creme dental com flúor com $28,1 \%$ ( $\mathrm{n}=20)$, o soro fisiológico com $23,9 \%$ $(\mathrm{n}=17)$ e $12,6 \%(\mathrm{n}=9)$ citaram a água destilada. 
Sobre a possibilidade da doença periodontal influenciar nas patologias sistêmicas, todos os acadêmicos consideravam que sim. Ao serem questionados se os patogênicos da cavidade bucal podem se disseminar para outros sistemas e contaminar outras regiões, 97,2\% ( $n=69)$ responderam que sim e 2,8\% $(n=2)$ não concordaram com essa afirmativa. Além disso, 66,19\% $(\mathrm{n}=47)$ dos graduandos manifestaram o desejo de trabalhar em UTI.

$\mathrm{Na}$ percepção dos acadêmicos, os principais pontos positivos em relação a atuação do cirurgião-dentista na UTI seriam proporcionar uma saúde bucal ao paciente com 90,1\% ( $\mathrm{n}=64)$, ter contato com uma equipe multidisciplinar com 85,9\% ( $\mathrm{n}=61$ ) e trabalhar em um ambiente de alta complexidade com 74,6\% ( $\mathrm{n}=53)$.

\section{Discussão}

Neste estudo, verificou-se que a maioria dos acadêmicos eram do sexo feminino e afirmaram ter algum conhecimento sobre a Odontologia Hospitalar, em consonância com o resultado obtido em estudo realizado em três universidades de Recife PE (Melo et al., 2017). As mulheres têm sido maioria no ensino superior e que apesar desses dados indicarem um avanço relacionado na atuação de mulheres na Odontologia, elas, ainda, possuem uma remuneração menor em relação aos homens que desenvolvem a mesma atividade (Costa, Durães \& Abreu, 2010).

A presença de um profissional em Odontologia (cirurgião-dentista) para avaliar as questões de saúde bucal nos pacientes da Unidade de Terapia Intensiva contribuem para saúde geral do paciente (Blum et al., 2017; Blum et al., 2018). Nesta pesquisa, todos os participantes afirmaram que o cirurgião-dentista deve ser integrante da equipe de UTI, resultado semelhante aos estudos realizados com acadêmicos de Odontologia do Amazonas - AM e cirurgiões-dentistas de São Paulo SP (Amaral et al., 2013; Ferreira, Ribeiro \& Santos, 2017).

No entanto, poucos se consideraram aptos para atuar nessa área na presente pesquisa. No Brasil, ainda existem poucas faculdades de Odontologia que possuem a disciplina Odontologia Hospitalar na grade curricular do curso, em alguns casos, a temática só é abordada em nível de especialização. Em contrapartida, algumas instituições incentivam atividades em ambiente hospitalar, como, por exemplo, projetos de extensão, permitindo que esses alunos tenham a oportunidade de vivenciar situações no âmbito hospitalar (Aranega, Bassi \& Ponzoni, 2012).

Em estudo realizado com 180 acadêmicos de Odontologia da Universidade Estadual da Paraíba (UEPB), 39\% acreditavam que a Odontologia Hospitalar é uma área pouco abordada ao decorrer da graduação. Enquanto $21 \%$ relataram como um conteúdo importante para a formação profissional, sendo abordado de forma fragmentada por várias disciplinas (Jordão, 2016). Assim fica clara a necessidade de maiores discussões relacionadas as matrizes curriculares para introduzir a Odontologia Hospitalar na formação acadêmica; o que contribuiria para formar um cirurgião-dentista com conhecimento sistêmico a comorbidades, tornando mais seguro o atendimento relacionado aos níveis de risco médico no seu campo de atuação.

Vale ressaltar que a Odontologia Hospitalar irá oferecer cuidados bucais a pacientes complexos internos no nível terciário de atenção e diante dessa complexidade dos pacientes, logo sugere-se uma formação em pós-graduação específica para a atuação em ambiente hospitalar.

De acordo com a resolução N²04/2019 do Conselho Federal de Odontologia, o profissional habilitado em Odontologia Hospitalar pode atuar em equipe multidisciplinar na promoção de saúde de modo a participar das decisões que incluem internação, diagnóstico, solicitação de exames e prescrição medicamentosa em conjunto com a equipe; prestar assistência odontológica em regime de internação hospitalar, ambulatorial, domiciliar e urgência, bem como atuar integrandose em programas de promoção, manutenção, prevenção, proteção e recuperação da saúde em ambiente hospitalar (Conselho Federal de Odontologia, 2019). 
Research, Society and Development, v. 10, n. 4, e15210413676, 2021

(CC BY 4.0) | ISSN 2525-3409 | DOI: http://dx.doi.org/10.33448/rsd-v10i4.13676

No presente estudo, o procedimento de solicitar exames complementares e realizar prescrições foi relatado com maior frequência pelos acadêmicos que tiveram a acesso a informações sobre Odontologia Hospitalar durante a graduação, com diferença significativa. Assim, ter acesso às informações sobre Odontologia Hospitalar na graduação podem contribuir para o esclarecimento dos acadêmicos sobre a atuação do cirurgião dentista em UTI.

Os cuidados odontológicos em pacientes atendidos na UTI colaboram na prevenção e restabelecimento do seu quadro sistêmico (Mattevi, 2011). Para tanto, de acordo com a Portaria SES - DF de No 287/2016, os principais procedimentos realizados pelos cirurgiões-dentistas na UTI são: exodontias, regularização de arestas dentais cortantes, suturas, confecção de protetores bucais, selamento de cavidades, raspagens para eliminação de placa dentária, diagnóstico de lesões patológicas e tratamento de lesões por meio farmacológico ou por laserterapia, assim como ações preventivas. As condutas citadas contribuem para redução de infecções respiratórias, e consequente taxa de mortalidade (Mattevi, 2011; Comissão Permanente de Protocolos de Atenção à Saúde da SES-DF, 2016).

No entanto, alguns alunos também relataram a realização de procedimentos como tratamento restaurador protético (21\%), tratamento endodôntico (33\%) e a exodontia de terceiros molares sem foco de infecção (31\%) e a frequência não mostrou diferença significativa de acordo com o fato de ter recebido ou não alguma aula na graduação sobre Odontologia Hospitalar. Esses procedimentos não são realizados em ambiente hospitalar, reforçando a necessidade da inserção da disciplina de Odontologia Hospitalar ou de mais carga horária para o assunto na graduação, pois, em alguns casos, o conteúdo é abordado em uma aula de um componente. Esse conhecimento insatisfatório dos profissionais em relação as patologias bucais e os cuidados bucais em UTI, é uma situação que merece atenção, principalmente no contexto informativo, educacional e clínico do cirurgião-dentista.

Foi observado que $66,19 \%(\mathrm{n}=47)$ dos acadêmicos consideraram o cirurgião-dentista como o responsável pela higiene bucal dos pacientes internos em UTI, enquanto 35,2\% (n=25) atribuiu à equipe de Enfermagem.

Na UTI, as práticas de higiene bucal, geralmente, são realizadas por enfermeiros. Um estudo preliminar realizado com enfermeiros e técnicos de Enfermagem, que atuam em hospitais públicos e particulares, evidenciou que apenas 30\% destes profissionais apresentavam conhecimento sobre as técnicas de escovação dentária (Araujo et al., 2009).

Associado a isso, um estudo realizado em 9 UTIs de três hospitais do Sul do Brasil indicou que 69,3\% da equipe de Enfermagem apresentou dificuldade para realizar a higiene bucal dos pacientes, no entanto, 22,1\% relatou não ter recebido o treinamento adequado para realizar essa tarefa (Blum et al., 2017). Em contrapartida, estudo realizado com profissionais de áreas ligadas diretamente a prática na UTI, como médicos, enfermeiros, cirurgiões-dentistas, fonoaudiálogos, nutricionistas e psicólogos demonstrou que $68,4 \%$ afirmaram ter recebido um treinamento regular em relação aos cuidados com a higiene bucal dos pacientes e 73,4\% das UTIs apresentavam um protocolo de higiene bucal definido (Blum et al., 2018).

Dessa forma, ressalta-se a importância do cirurgião-dentista atuar em conjunto com a equipe de enfermagem nas instruções e orientações sobre os protocolos de higiene bucal para que o protocolo de atuação da odontologia seja reconhecido pelos profissionais e que a equipe de enfermagem possa desempenhar a higiene bucal adequada.

Quanto ao produto utilizado nos protocolos de higiene bucal, $98,5 \%$ responderam a Clorexidina a $0,12 \%$ como o mais utilizado. Neste contexto, a Associação de Medicina Intensiva Brasileira (AMIB), preconiza a utilização da Clorexidina a $0,12 \%$ para a realização da higiene bucal dos pacientes, buscando a prevenção de casos de pneumonia associada à ventilação mecânica, como apresentado no Procedimento Operacional Padrão - POP (Brasil, 2014).

Para casos de pacientes com a Covid-19, a AMIB recomenda-se bochechos com Peróxido de Hidrogênio a 1\% antes de cada atendimento, pois o vírus SARS-CoV-2 é vunerável a oxidação (Brasil, 2020). 
Nesta pesquisa, os participantes afirmaram que a doença periodontal pode influenciar nas patologias sistêmicas (100\%). Estudo transversal, realizado na UTI de um hospital em Belo Horizonte - MG, constatou a prevalência de periodontite em pacientes internados em UTI, além disso, observou uma associação positiva entre a periodontite e as doenças cardiovasculares (Albuquerque et al., 2018). Essa condição pode contribuir para o aumento no custo do tratamento hospitalar e no tempo de internação, além de impactar na qualidade de vida dos pacientes.

As transformações que vêm ocorrendo na sociedade e na educação, bem como a complexidade que tem marcado a contemporaneidade, encontra-se a questão da fragmentação do conhecimento. O ensino especializado vem fragmentando em unidades estanques, o ambiente universitário. $\mathrm{O}$ grande desafio imposto pela interdisciplinaridade é a necessidade de uma mudança de atitude que possibilite ao indivíduo conhecer realmente os limites de seu saber para que ele consiga acolher contribuições de outras disciplinas. A interdisciplinaridade deve ser entendida, antes de tudo, como uma atitude necessária para interpretar corretamente as informações e contextualizá-las (Couto, 2011).

Os resultados demonstraram que os acadêmicos consideraram a necessidade do cirurgião-dentista na equipe multidisciplinar e que reconheceram a atuação desses profissionais no ambiente hospitalar, bem como, algumas informações sobre a prática em ambiente hospitalar. No entanto, relataram a baixa frequência da discussão de Odontologia Hospitalar e de atividades em ambiente hospitalar durante a graduação.

\section{Conclusão}

Portanto, o presente estudo mostrou a percepção dos acadêmicos de Odontologia sobre a atuação do cirurgião-dentista no ambiente hospitalar e que esses futuros profissionais reconheceram a sua importância na equipe multidisciplinar da UTI, com o intuito de prevenir intercorrências e infecções hospitalares, assim como garantir a manutenção da saúde bucal e da qualidade de vida dos pacientes internos, minimizando possíveis complicações.

\section{Referências}

Albuquerque, B. N., Araújo, M. M., Silva, T. A., Cota, L. O. M., Cortelli, S. C. \& Costa, F. O. (2018). Periodontal Condition and Immunological Aspects of Individuals Hospitalized in the Intensive Care Unit. Brazilian Dental Journal, 29(3):301-308.

Amaral, C. O. F., Marques, J. A., Bovolato, C. M., Parizi, A. G. S., Oliveira, A. \& Stratioto, F. G. (2013). Importância do Cirurgião-dentista em Unidade de Terapia Intensiva: avaliação multidisciplinar. Revista da Associacao Paulista de Cirurgiões Dentistas, 67(2):107-11.

Aranega, A. M., Bassi, A. P. F., Ponzoni, D., Wayama, T. M., Esteves, J. C. \& Junior, I. R. G. (2012). Qual a importância da Odontologia Hospitalar?. Revista Brasileira de Odontologia, 69(1):90-3.

Araújo, R. J. G., Oliveira, L. C. G., Hanna, L. M. O., Canêa, A. M. \& Álvares, N. N. C. F. (2009). Análise de percepções e ações de cavidades bucais realizados por equipes de enfermagem em unidades de terapia intensiva. Revista Brasileira de Terapia Intensiva, 21(1):38-44.

Blum, D. F. C, Munaretto, J., Baeder, F. M., Gomez, J., Castro, C. P. P. \& Bona, Á. D. (2017). Influence of dentistry professionals and oral health assistance protocols on intensive care unit nursing staff. A survey study. Revista Brasileira de Terapia Intensiva, 29(3):391-393.

Blum, D. F. C., Silva, J. A. S., Baeder, F. M. \& Bona, Á. D. (2018). A atuação da Odontologia em unidades de terapia intensiv a no Brasil. Revista Brasileira de Terapia Intensiva, 30(3):327-332.

Brasil, D. O. D. E. (2014). Departamento de Odontologia e Departamento de Enfermagem. Recomendações para higiene bucal do paciente em UTI. Associação de Medicina Intensiva Brasileira - AMIB. São Paulo.

Brasil, A. M. I. B. (2020). Associação de Medicina Intensiva Brasileira. Recomendações AMIB/CFO para atendimento odontológico COVID-19: Comitê de Odontologia AMIB/CFO de enfrentamento ao COVID-19 Departamento de Odontologia AMIB.

Brasil, C. D. D. (2008). Câmarâ dos Deputados. Projeto de lei $\mathrm{n}^{\circ} 2.776$ de abril de 2008. Dispõe sobre a obrigatoriedade da presença de profissionais de odontologia na Unidade de Terapia Intensiva.

Couto, R. M. S. (2011). Fragmentação do conhecimento ou interdisciplinaridade: ainda um dilema contemporâneo? Revista faac, 1(1):11-19.

Costa, S. M., Durães, S. J. A. \& Abreu, M. H. N. G. (2010). Feminização do curso de odontologia da Universidade Estadual de Montes Claros. Revista Ciência \& Saúde Coletiva, 15(1):1865-1873. 
Research, Society and Development, v. 10, n. 4, e15210413676, 2021

(CC BY 4.0) | ISSN 2525-3409 | DOI: http://dx.doi.org/10.33448/rsd-v10i4.13676

Comissão Permanente de Protocolos de Atenção à Saúde da SES-DF - CPPAS (2016). Protocolo de Atenção à Saúde: Atendimento Odontológico em UTI.

De Odontologia, C. F. (2019). Conselho Federal de Odontologia. 204: RESOLUÇÃO CFO-204, de 21 de maio de 2019. Brasília, $2: 12$.

Ferreira, L. S., Ribeiro, E. O. A. \& Santos, R. E. A. (2017). Conhecimentos dos acadêmicos de odontologia da UEA sobre a odon tologia hospitalar. Revista da Academia Brasileira de Odontologia, 26(1):38-43.

Jordão, T. F. (2016). Conhecimento de acadêmicos de Odontologia sobre a atuação do cirurgião dentista no contexto hospitalar. (Monografia de graduação). Araruna: Universidade Estadual da Paraíba - UEPB.

Marshall, J. C., Bosco, L., Adhikari, N. K., Connolly, B., Diaz, J. V., Dorman, T., Fowler, R. A., Meyfroidt, G., Nakagawa, S., Pelosi, P., Vincent, J. L., Wollman, K. \& Zimmerman, J. (2017). What is an intensive care unit? A report of the task force of the World Federation of Societies of Intensive and Critical Care Medicine. Journal of critical care, 37: 270-276.

Morais, T. M. N., Silva, A., Avi, A. L. R. O., Souza, P. H. R., Knobel, E. \& Camargo, L. F. A. (2008). A importância da atuação odontológica em pacientes internados em unidade de terapia intensiva. Revista Brasileira de Terapia Intensiva, 18(4):412-7.

Melo, A. R. S., Sales, T. F. L. L., Melo, A. C. M., Lins, C. C. S. A. \& Zimmermann, R. D. (2017). Percepção de ações e cuidad os bucais em unidade de tratamento intensivo por estudantes de odontologia. Odontologia Clínico-Científica (Online), 16(2):127-134.

Mattevi, G. S., Figuereido, D. R., Patrício, Z. M. \& Rath, I. B. S. (2011). Participação do cirurgião-dentista em equipe de saúde multidisciplinar na atenção à saúde da criança no contexto hospitalar. Revista Ciência \& Saúde Coletiva, 16(10):4229-36.

Ory, J., Mourgues, C., Raybaud, E., Chabanne, R., Jourdy, J. C., Belard, F., Guérin, R., Cosserant, B., Faure, J. S., Calvet, L., Pereira, B., Guelon, D., Traore, O. \& Gerbaud, L. (2018). Cost assessment of a new oral care program in the intensive care unit to prevent ventilator-associated pneumonia. Clinical Oral Investigations, 22(5):1945-1951.

Pereira A. S.; Shitsuka D. M.; Parreira F. J. \& Shitsuka R. (2018). Metodologia da pesquisa científica. UFSM.

Sampaio-Maia, B., Caldas, I. M., Pereira, M. L., Pérez-Mongiovi, D. \& Araujo, R. (2016). The oral microbiome in health and its implication in oral and systemic diseases. Advances in Applied Microbiology, 97:171-210. 